\title{
CHARACTERIZATION AND UNIQUENESS OF NONLINEAR UNIFORM APPROXIMATION
}

\author{
by WENSHAN YANG, CHONG LI and G. A. WATSON
}

(Received 10th August 1995)

\begin{abstract}
Characterization and uniqueness results are given for uniform approximation which extend to suns previously known results for convex sets.
\end{abstract}

1991 Mathematics subject classification: 41 A50.

\section{Introduction}

Let $X$ be a compact Hausdorff space, and let $C(X)$ be the Banach space of continuous real-valued functions defined on $X$ with the uniform norm

$$
\|f\|=\max _{x \in X}|f(x)|
$$

Let $G$ be a non-empty subset of $C(X)$, and let $f \in C(X) \backslash \bar{G}$. Then we are concerned here with the problem of finding a best approximation $g_{0} \in G$ to $f$ in the sense that

$$
\left\|f-g_{0}\right\|=\inf _{g \in G}\|f-g\| \text {. }
$$

Such a $g_{0}$ is called a best approximation to $f$ from $G$, and the set of all best approximations is denoted by $P_{G}(f)$.

An important role in the characterization of best approximations is played by the Kolmogorov Criterion. For any $h \in C(X)$, let

$$
X_{h}=\{x \in X:|h(x)|=\|h\|\} .
$$

Definition 1. The pair $f \in C(X), g_{0} \in G$ satisfy the Kolmogorov Criterion (KC) if

$$
\max _{x \in X_{f-\infty}}\left(f-g_{0}\right)(x)\left(g_{0}-g\right)(x) \geq 0, \quad \text { for all } g \in G .
$$

If strict inequality holds for all $g \in G \backslash\left\{g_{0}\right\}$, then $\left(f, g_{0}\right)$ is said to satisfy the Strict Kolmogorov Criterion (SKC). 
For brevity in what follows, we will use the expression " $\left(f, g_{0}\right)$ satisfies (KC)" to mean that $\left(f, g_{0}\right)$ satisfies the Kolmogorov Criterion, and this convention will be used in other (analogous) situations. For any $G$, a sufficient condition for $g_{0} \in P_{G}(f)$, where $f \in C(X)$, is that $\left(f, g_{0}\right)$ satisfies (KC), and if $G$ is convex, this condition is also necessary and so a complete characterization is provided. In fact this extends beyond convex sets through the concept of a sun.

Definition 2. A subset $G$ of $C(X)$ is a sun of $C(X)$ if for each $f \in C(X), g_{0} \in P_{G}(f)$ implies that $g_{0} \in P_{G}\left(g_{0}+t\left(f-g_{0}\right)\right)$ for all $t>0$.

It may be shown that the following are equivalent

(1) $G$ is a sun

(2) $g_{0} \in P_{G}(f)$, where $f \in C(X)$, if and only if $\left(f, g_{0}\right)$ satisfies (KC).

This characterization of suns was in fact established for general normed linear spaces (with suitable generalization of the Kolmogorov Criterion) by Brosowski [3], who, with co-workers, developed in the 60's a theory for such sets analogous to the linear or convex theory (see Amir and Deutch [1] for some references, and also some further properties). Related results are also due to Vlasov (for example [10]).

A sufficient condition for $P_{G}(f)=\left\{g_{0}\right\}$ is that $\left(f, g_{0}\right)$ satisfies (SKC). However even for convex sets it is well known that this condition is not necessary. In [7] Nurnberger proves that if $G$ is a finite dimensional convex subset of $C(X)$, then the following are equivalent:

(1) $G$ is a semi-Chebyshev subset of $C(X)$ (that is, every $f \in C(X)$ has at most one best approximation in $G$ )

(2) for each $f \in C(X) \backslash \bar{G}, g_{0} \in G, g_{0} \in P_{G}(f)$ if and only if $\left(f, g_{0}\right)$ satisfies (SKC).

This result was recently extended to a class of generalized constrained rationals in [4], but it is clear that the techniques used in [4] or [7] do not extend to more general subsets of $C(X)$. Here, a different approach is used to permit extension to suns of $C(X)$. This is considered in Section 3 , and the specific result is stated as Corollary 3.1 .

Analogous conditions to those given in Definition 1 which can be used to characterize best and unique best approximations are due to Papini [8].

Definition 3. The pair $f \in C(X), g_{0} \in G$ satisfy the Papini Criterion (PC) if

$$
\max _{x \in X_{f-g}}(f-g)(x)\left(g-g_{0}\right)(x) \leq 0, \quad \text { for all } g \in G
$$

If strict inequality holds for all $g \in G \backslash\left\{g_{0}\right\}$, then $\left(f, g_{0}\right)$ is said to satisfy the Strict Papini Criterion (SPC). 
For any $G$, a necessary condition for $g_{0} \in P_{G}(f)$, where $f \in C(X)$, is that $\left(f, g_{0}\right)$ satisfies (PC), and if $G$ is convex, it is proved in Papini [8] that this condition is also sufficient. A necessary condition for $P_{G}(f)=\left\{g_{0}\right\}$ is that $\left(f, g_{0}\right)$ satisfies (SPC), and it is also shown in [8] that if $G$ is convex this too is sufficient. It has been an open question whether or not these characterizations extend to suns, and an affirmative answer is given here. This is given in the following section as Theorem 2.1.

\section{The Papini Criterion and characterization}

A useful concept in what follows is that of the weak betweeness property (sometimes called the closed sign property).

Definition 4. A subset $G$ of $C(X)$ has the weak betweeness property if for any two distinct elements $g_{0}, g_{1}$ of $G$, and a closed subset $D$ of $X$ with $\min _{x \in D}\left|g_{1}(x)-g_{0}(x)\right|>0$, there exists a sequence $\left\{g_{n}\right\} \subset G$ such that

$$
\begin{gathered}
\left\|g_{n}-g_{0}\right\| \rightarrow 0, \\
\min _{x \in D}\left(g_{1}-g_{0}\right)(x)\left(g_{n}-g_{0}\right)(x)>0 \quad n=2,3, \ldots
\end{gathered}
$$

This property is a sufficient property for best approximations to be characterized by the extrema of their error function (see Dunham [5]). It includes the so-called betweeness property, due to Dunham [6]. In fact it is shown by Braess [2] (note that Braess refers to a sun as defined here as a strict sun) that the following are equivalent:

(1) $G$ is a sun,

(2) $G$ has the weak betweeness property.

This equivalence can be used to prove the following lemma.

Lemma 2.1. Let $G$ be a sun of $C(X)$, and let $f \in C(X) \backslash \bar{G}, g_{0} \in G$. Then if

$$
\left(f, g_{0}\right) \text { satisfies (PC) }
$$

it follows that

$$
\left(f, g_{0}\right) \text { satisfies }(\mathrm{KC}) \text {. }
$$

Proof. Let the conditions hold, let $\left(f, g_{0}\right)$ satisfy (PC) but suppose that $\left(f, g_{0}\right)$ does not satisfy $(\mathrm{KC})$. Then there exists $g \in G$ such that

$$
\max _{x \in X_{f-\infty}}\left(f-g_{0}\right)(x)\left(g_{0}-g\right)(x)<-\delta<0 .
$$


Now it is well known that for any $f, g \in C(X)$

$$
\lim _{t \rightarrow 0+} \frac{\|f+t g\|-\|f\|}{t}=\max _{x \in X_{f}}(\operatorname{sign} f(x)) g(x) .
$$

Further, it is shown in [9] that

$$
\lim _{t \rightarrow 0+} \frac{\|f+t g\|-\|f\|}{t}=\lim _{\ell \rightarrow 0+} \sup _{x \in X_{(, \ell)}}(\operatorname{sign} f(x)) g(x),
$$

where for $\varepsilon>0$,

$$
X_{(, e)}=\{x \in X:|f(x)| \geq\|f\|-\varepsilon\}
$$

Therefore it follows that

$$
\lim _{\varepsilon \rightarrow 0+} \max _{x \in X_{(J-80, \varepsilon)}}\left(f-g_{0}\right)(x)\left(g_{0}-g\right)(x)<-\delta .
$$

Thus there exists $\varepsilon_{0}>0$ such that for any $\varepsilon \in\left(0, \varepsilon_{0}\right]$

$$
\max _{x \in X_{\left(J-0_{0}, \varepsilon\right)}}\left(f-g_{0}\right)(x)\left(g_{0}-g\right)(x)<-\delta .
$$

Let $\varepsilon_{1}=\min \left\{\varepsilon_{0},\left\|f-g_{0}\right\| / 4\right\}, D=X_{\left(f-g_{0}, e_{1}\right)}$. Then we have

$$
\begin{gathered}
\operatorname{sign}\left(f-g_{0}\right)(x)=\operatorname{sign}\left(g-g_{0}\right)(x) \quad \text { for all } x \in D, \\
\min _{x \in D}\left|g(x)-g_{0}(x)\right|>0 .
\end{gathered}
$$

Since $G$ is a sun, it has the weak betweeness property, and so there exists a sequence $\left\{g_{n}\right\} \subset G$ such that

$$
\begin{gathered}
\left\|g_{n}-g_{0}\right\| \rightarrow 0 \\
\min _{x \in D}\left(g-g_{0}\right)(x)\left(g_{n}-g_{0}\right)(x)>0, \quad n=2,3, \ldots
\end{gathered}
$$

Now let $N$ be large enough that

$$
\left\|g_{n}-g_{0}\right\|<\varepsilon_{1} / 3, \quad n>N .
$$

Since for each $n>N$, and $x \in D$,

$$
\begin{aligned}
\left|f(x)-g_{0}(x)\right| & \geq\left\|f-g_{0}\right\|-\varepsilon_{1} \\
& \geq \frac{3}{4}\left\|f-g_{0}\right\| \\
& >\left\|g_{n}-g_{0}\right\|,
\end{aligned}
$$


it follows that

$$
\left(\left(f-g_{0}\right)(x)\right)\left(f-g_{n}\right)(x)=\left(\left(f-g_{0}\right)(x)\right)^{2}+\left(f-g_{0}\right)(x)\left(g_{0}-g_{n}\right)(x)>0 .
$$

Thus if $n>N$,

$$
\operatorname{sign}\left(f-g_{0}\right)(x)=\operatorname{sign}\left(f-g_{n}\right)(x) \text { for all } x \in D .
$$

Now for each $x \in X_{f-g_{n}}$, and $n>N$,

$$
\begin{aligned}
\left|f(x)-g_{0}(x)\right| & \geq\left|f(x)-g_{n}(x)\right|-\left|g_{n}(x)-g_{0}(x)\right| \\
& \geq\left\|f-g_{0}\right\|-2\left\|g_{n}-g_{0}\right\| \\
& \geq\left\|f-g_{0}\right\|-\frac{2}{3} \varepsilon_{1} \\
& \geq\left\|f-g_{0}\right\|-\varepsilon_{1} .
\end{aligned}
$$

It follows that $X_{f-g_{n}} \subset D$ for all $n>N$.

Now using (2.1), (2.4), and (2.5) it follows that for all $n>N$

$$
\begin{aligned}
\max _{x \in X_{f-g_{n}}}\left(\operatorname{sign}\left(f-g_{n}\right)(x)\right)\left(g_{n}-g_{0}\right)(x) & \geq \min _{x \in X_{f-g_{n}}}\left(\operatorname{sign}\left(f-g_{n}\right)(x)\right)\left(g_{n}-g_{0}\right)(x) \\
& \geq \min _{x \in D}\left(\operatorname{sign}\left(f-g_{0}\right)(x)\right)\left(g_{n}-g_{0}\right)(x) \\
& =\min _{x \in D}\left(\operatorname{sign}\left(g-g_{0}\right)(x)\right)\left(g_{n}-g_{0}\right)(x) \\
& >0 .
\end{aligned}
$$

This contradicts the fact that $\left(f, g_{0}\right)$ satisfies (PC), and completes the proof.

Corollary 2.1. Let $G$ be a subset of $C(X)$. Then the following statements are equivalent:

(1) $G$ is a sun of $C(X)$.

(2) for any $f \in C(X) \backslash \bar{G}, g_{0} \in G$, then $\left(f, g_{0}\right)$ satisfies (PC) implies $\left(f, g_{0}\right)$ satisfies (KC).

Proof. The fact that (2) is implied by (1) is a direct consequence of Lemma 2.1, so it remains to prove the reverse implication. Assume (2). For any $f \in C(X) \backslash \bar{G}$, $g_{0} \in P_{G}(f),\left(f, g_{0}\right)$ satisfies (PC), and so by (2), $\left(f, g_{0}\right)$ satisfies $(\mathrm{KC})$. In other words $g_{0} \in P_{G}(f)$ if and only if $\left(f, g_{0}\right)$ satisfies (KC). The result follows.

We are now in a position to prove the main result of this section. 
Theorem 2.1. Let $G$ be a sun of $C(X)$. For $f \in C(X) \backslash \bar{G}, g_{0} \in G$, then

(i) $g_{0} \in P_{G}(f)$ if and only if $\left(f, g_{0}\right)$ satisfies (PC).

(ii) $P_{G}(f)=\left\{g_{0}\right\}$ if and only if $\left(f, g_{0}\right)$ satisfies (SPC).

Proof. The proof of (i) follows from Lemma 2.1, so consider (ii). Suppose that $\left(f, g_{0}\right)$ satisfies (SPC). Then by (i), $g_{0} \in P_{G}(f)$. Let there exist another $g_{1} \in P_{G}(f)$. Then $\left(f, g_{1}\right)$ satisfies (PC), and so $\left(f, g_{1}\right)$ satisfies (KC), by Lemma 2.1. Thus

$$
\max _{x \in X_{f-g_{1}}}\left(f-g_{1}\right)(x)\left(g_{1}-g_{0}\right)(x) \geq 0
$$

which contradicts the assumption that $\left(f, g_{0}\right)$ satisfies (SPC). The reverse implication is immediate, and the proof is complete.

Arguing as in [4, Corollary 1$]$, the following corollary is obtained.

Corollary 2.2. Let the assumptions of Theorem 2.1 hold. Then

(1) $g_{0} \in P_{G}(f)$ if and only if

$$
\max _{x \in X_{f-g}}(f-g)(x)\left(g-g_{0}\right)(x) \leq \max _{x \in X_{f-g_{0}}}\left(f-g_{0}\right)(x)\left(g_{0}-g\right)(x), \quad \text { for all } g \in G,
$$

(2) $P_{G}(f)=\left\{g_{0}\right\}$ if and only if

$$
\max _{x \in X_{f-g}}(f-g)(x)\left(g-g_{0}\right)(x)<\max _{x \in X_{f-\infty}}\left(f-g_{0}\right)(x)\left(g_{0}-g\right)(x), \quad \text { for all } g \in G \backslash\left\{g_{0}\right\} .
$$

\section{The Strict Kolmogorov Criterion and uniqueness}

An important role in what follows is played by the concept of a uniqueness element.

Definition 5. Let $G \subset C(X)$. Then $g_{0}$ is said to be a uniqueness element of $G$ if for any $f \in C(X), g_{0} \in P_{G}(f)$ implies $P_{G}(f)=\left\{g_{0}\right\}$.

A useful consequence of $g_{0}$ being a uniqueness element is given in the following lemma.

Lemma 3.1. Let $G \subset C(X), g_{0} \in G$ be a uniqueness element of $G$. Then for any $h \in G, h \neq g_{0}$, any closed subset $Z$ of zeros of $g_{0}-h$, and any $s \in C(X)$ with $\|s\| \leq 1$, taking the values +1 or -1 on $Z$, there exists $p \in G$ such that

$$
\operatorname{sign}\left(p-g_{0}\right)(x)=s(x), \quad \text { for all } x \in Z
$$


Proof. For any $h \neq g_{0}, h \in G$, and any closed subset $Z$ of zeros of $h-g_{0}$, let $s \in C(X),\|s\| \leq 1$, such that $|s(x)|=1$ for $x \in Z$. Define $f$ by

$$
f(x)=g_{0}(x)+s(x)\left[\left\|g_{0}-h\right\|-\left|g_{0}(x)-h(x)\right|\right], \quad \text { for all } x \in X
$$

It follows that

$$
\left\|f-g_{0}\right\|=\left\|g_{0}-h\right\|
$$

and

$$
Z \subset X_{f-g_{0}}
$$

For all $x \in X$,

$$
\begin{aligned}
|(f-h)(x)| & \leq\left|\left(f-g_{0}\right)(x)\right|+\left|\left(g_{0}-h\right)(x)\right| \\
& \leq\left\|g_{0}-h\right\| \\
& =\left\|f-g_{0}\right\| .
\end{aligned}
$$

Thus $g_{0} \in P_{G}(f)$ implies that $h \in P_{G}(f)$, which is a contradiction that $g_{0}$ is a uniqueness element. Thus $g_{0} \notin P_{G}(f)$. It follows that there exists $p \in G$ such that

$$
\|f-p\|<\left\|f-g_{0}\right\|,
$$

which implies that

$$
|(f-p)(x)|<\left|\left(f-g_{0}\right)(x)\right|, \quad \text { for all } x \in Z
$$

Thus

$$
\left(f-g_{0}\right)(x)\left(p-g_{0}\right)(x)>0, \quad \text { for all } x \in Z
$$

Obviously,

$$
\operatorname{sign}\left(f-g_{0}\right)(x)=s(x), \quad \text { for all } x \in Z
$$

and so

$$
\operatorname{sign}\left(p-g_{0}\right)(x)=s(x), \quad \text { for all } z \in Z
$$

and the proof is complete.

We can now characterize a uniqueness element of a sun of $C(X)$ in terms of the Strict Kolmogorov Criterion. 
Theorem 3.2. Let $G$ be a sun of $C(X)$. Then the following statements are equivalent:

(1) $g_{0} \in G$ is a uniqueness element of $G$

(2) $f \in C(X) \backslash \bar{G}, g_{0} \in P_{G}(f)$ if and only if $\left(f, g_{0}\right)$ satisfies (SKC).

Proof. The proof that (2) implies (1) is direct, so we consider the proof that (1) implies (2). Assume (1) holds. Sufficiency in (2) is immediate, so assume that for some $f \in C(X) \backslash \bar{G}, g_{0} \in P_{G}(f)$ there exists $g_{1} \in G, g_{1} \neq g_{0}$ such that

$$
\max _{x \in X_{f-g_{0}}}\left(f-g_{0}\right)(x)\left(g_{0}-g_{1}\right)(x) \leq 0 \text {. }
$$

Since $G$ is a sun of $C(X)$, and $g_{0} \in P_{G}(f)$, then $(\mathrm{KC})$ is satisfied so that

$$
\max _{x \in X_{f-g_{0}}}\left(f-g_{0}\right)(x)\left(g_{0}-g\right)(x) \geq 0, \quad \text { for all } g \in G .
$$

From (3.1) and (3.2), it follows that

$$
\max _{x \in X_{f-\infty}}\left(f-g_{0}\right)(x)\left(g_{0}-g_{1}\right)(x)=0
$$

Now define $N$ by

$$
N=\left\{x \in X_{f-g_{0}}: g_{0}(x)=g_{1}(x)\right\}
$$

Clearly $N$ is non-empty. Let $s \in C(X)$ satisfy $\|s\| \leq 1$ and

$$
s(x)=\operatorname{sign}\left(f(x)-g_{0}(x)\right), \quad x \in N \text {. }
$$

Then with this function $s$, and taking $h=g_{1}$, it follows from Lemma 3.1 that for some $p \in G$

$$
\left(f-g_{0}\right)(x)\left(p-g_{1}\right)(x)>0, \quad \text { for all } x \in N,
$$

using the fact that $g_{0}(x)=g_{1}(x)$ on $N$. By the continuity of $\left(f-g_{0}\right)(x)\left(p-g_{0}\right)(x)$ on $X_{f-g_{0}}$, there exists a subset $U^{\prime}$ of $X_{f-g_{0}}$, open with respect to $X_{f-g_{0}}$, on which (3.4) holds. Now there exists a subset $U$ of $U^{\prime}$, open with respect to $X_{f-g_{0}}$, such that

$$
N \subset U \subset \bar{U} \subset U^{\prime} \subset X_{f-80}
$$

Since $G$ has the weak betweeness property there exists a sequence $\left\{g_{n}\right\} \subset G$ converging uniformly to $g_{1}$, such that

$$
\left(g_{1}-g_{n}\right)(x)\left(g_{1}-p\right)(x)>0, \text { for all } x \in \bar{U}
$$


Since (3.4) holds on $\bar{U}$ it follows that

$$
\left(f-g_{0}\right)(x)\left(g_{n}-g_{1}\right)(x)>0, \quad \text { for all } x \in \bar{U} .
$$

Further, $V=X_{f-g_{0}} \backslash U$ is closed and contains no points of $N$, and so by (3.1),

$$
\left(f-g_{0}\right)(x)\left(g_{1}-g_{0}\right)(x)>0, \quad \text { for all } x \in V .
$$

Because $g_{n}$ converges uniformly to $g_{1}$, for all $n$ large enough

$$
\left(f-g_{0}\right)(x)\left(g_{n}-g_{0}\right)(x)>0, \quad \text { for all } x \in V .
$$

From (3.5) and (3.6), it follows that for $n$ large enough

$$
\left(f-g_{0}\right)(x)\left(g_{0}-g_{n}\right)(x)<0, \quad \text { for all } x \in X_{f-g_{0}} .
$$

This contradicts (3.2), and the result is proved.

The main result of this section is now an easy corollary of this theorem.

Corollary 3.1. Let $G$ be $a$ sun of $C(X)$. Then the following statements are equivalent:

(1) $G$ is a semi-Chebyshev subset of $C(X)$

(2) for any $f \in C(X) \backslash \bar{G}, g_{0} \in G, g_{0} \in P_{G}(f)$ if and only if $\left(f, g_{0}\right)$ satisfies (SKC).

Acknowledgement. The work of the first two authors was supported by the National Natural Science Foundation of China.

\section{REFERENCES}

1. D. AmIR and F. Deutch, Suns, moons and quasi-polyhedra, J. Approx. Theory 6 (1972), 176-201.

2. D. BRAESS, Nonlinear Approximation Theory (Springer Verlag, Berlin, 1986).

3. B. BrosowsKI, Nichtlineare Approximation in normierten Vectorraumen, in Abstract Spaces and Approximation (eds. P. L. Butzer and B. Sz-Nagy, ISNM 10, Birkhauser, Basel, 1969), 140-159.

4. Li Chong and G. A. Watson, Characterization of a best and a unique best approximation from constrained rationals, Comput. Math. Appl. 30 (1995), 51-57.

5. C. B. Dunham, Characterizability and uniqueness in real Chebyshev approximation, $J$. Approx. Theory 2 (1969), 374-383.

6. C. B. Dunham, Chebyshev approximation by families with the betweeness property, Trans. Amer. Math. Soc. 136 (1969), 151-157.

7. G. NURNBERGER, Unicity and strong unicity in approximation theory, J. Approx. Theory 26 (1979), 54-70. 
8. P. L. PaPINI, Approximation and norm derivatives in real normed spaces, Resultate Math. 5 (1982), 81-94.

9. K. SATo, On the generators of non-negative contraction semi-groups in Banach spaces, $J$. Math. Soc. Japan 20 (1968), 420-436.

10. L. P. Vlasov, Chebyshev sets in Banach Spaces, Soviet Math. Dokl. 2 (1961), 13731374.

RESEARCH BUREAU

ZHEJIANG NORMAL UNIVERSITY

JiNHUA CiTY

ZHEJIANG

P.R. China
Department of Mathematics

HaNGzhou INSTITUTE OF COMMERCE

HANGZHOU

P.R. China

Department of Mathematics and Computer Science

UNIVERSITY OF DUNDEE

DUNDEE DD1 4HN

SCOTLAND

E-mail address: gawatson@mcs.dundee.ac.uk 